\title{
Complex Coding and Regulatory Polymorphisms in a Restriction Factor Determine the Susceptibility of Drosophila to Viral Infection
}

\author{
Chuan Cao, ${ }^{*, 1}$ Rodrigo Cogni, ${ }^{*, 2}$ Vincent Barbier, ${ }^{+, 3}$ and Francis M. Jiggins* \\ *Department of Genetics, University of Cambridge, CB2 3EH, United Kingdom, ${ }^{\dagger}$ Institut de Biologie Moléculaire et Cellulaire, \\ Centre National de la Recherche Scientifique, Strasbourg, 67084, France
}

\begin{abstract}
It is common to find that major-effect genes are an important cause of variation in susceptibility to infection. Here we have characterized natural variation in a gene called pastrel that explains over half of the genetic variance in susceptibility to the Drosophila C virus (DCV) in populations of Drosophila melanogaster. We found extensive allelic heterogeneity, with a sample of seven alleles of pastrel from around the world conferring four phenotypically distinct levels of resistance. By modifying candidate SNPs in transgenic flies, we show that the largest effect is caused by an amino acid polymorphism that arose when an ancestral threonine was mutated to alanine, greatly increasing resistance to DCV. Overexpression of the ancestral, susceptible allele provides strong protection against DCV; indicating that this mutation acted to improve an existing restriction factor. The pastrel locus also contains complex structural variation and cis-regulatory polymorphisms altering gene expression. We find that higher expression of pastrel is associated with increased survival after DCV infection. To understand why this variation is maintained in populations, we investigated genetic variation surrounding the amino acid variant that is causing flies to be resistant. We found no evidence of natural selection causing either recent changes in allele frequency or geographical variation in frequency, suggesting that this is an old polymorphism that has been maintained at a stable frequency. Overall, our data demonstrate how complex genetic variation at a single locus can control susceptibility to a virulent natural pathogen.
\end{abstract}

KEYWORDS Drosophila; DCV; pastrel; viral infection; natural variation; Genetics of immunity

central aim of infectious disease research is to understand why individuals within populations vary in their susceptibility to infection. This variation often has a substantial genetic component, and much effort has been devoted to identifying the genes involved [see reviews on humans (Burgner et al. 2006), plants (Alonso-Blanco and Mendez-Vigo 2014), and invertebrates (Obbard and Dudas 2014)]. It is

Copyright $\odot 2017$ Cao et al.

doi: https://doi.org/10.1534/genetics.117.201970

Manuscript received March 17, 2017; accepted for publication June 12, 2017; published Early Online June 19, 2017.

Available freely online through the author-supported open access option.

This is an open-access article distributed under the terms of the Creative Commons Attribution 4.0 International License (http://creativecommons.org/icenses/by/4.0/, which permits unrestricted use, distribution, and reproduction in any medium, provided the original work is properly cited.

Supplemental material is available online at www.genetics.org/lookup/suppl/doi:10. 1534/genetics.117.201970/-/DC1.

${ }^{1}$ Corresponding author: Wellcome Trust Sanger Institute, Wellcome Trust Genome Campus, Hinxton, Cambridge, CB10 1SA, United Kingdom. E-mail: chuan.cao@sanger. ac.uk

${ }^{2}$ Present address: Department of Ecology, University of São Paulo, São Paulo 05508900, Brazil.

${ }^{3}$ Present Address: Institute for Immunology and Informatics, University of Rhode Island, Providence, RI 02903. common to find that natural populations contain major-effect polymorphisms that affect susceptibility to infection, especially when natural pathogens or parasites are studied. In humans, for example, major-effect genes affect susceptibility to Plasmodium falciparum malaria, $P$. vivax malaria, HIV, and Norwalk virus diarrhea (Hill 2012). Studying these genes can not only advance our understanding of the mechanisms of resistance and functioning of immune systems, but also provide insights into evolutionary processes. For example, theoretical models of host-parasite coevolution make strong assumptions about the genetic basis of resistance (Routtu and Ebert 2015). More generally, pathogens are one of the most important selective agents in nature, so understanding the genetic basis of how host populations respond to this selection pressure is of great interest.

While much research has focused on humans, crops, and domestic animals, studying the natural pathogens of model organisms such as Arabidopsis, Drosophila, and Caenorhabditis elegans provides a powerful way to understand the genetics of infectious disease resistance. There has been substantial research into genetic variation in susceptibility to viruses in 
Drosophila, with most research focusing on the sigma virus (Rhabdoviridae; DMelSV) (Longdon et al. 2012) and Drosophila C virus (DCV) (Dicistroviridae) (Johnson and Christian 1998; Hedges and Johnson 2008; Magwire et al. 2012; Kemp et al. 2013; Longdon et al. 2013; Zhu et al. 2013; Ferreira et al. 2014; Martins et al. 2014). DMelSV is a vertically transmitted virus that is relatively benign, causing an $\sim 20 \%$ drop in fitness (Yampolsky et al. 1999; Wilfert and Jiggins 2013). In contrast, DCV is horizontally transmitted and multiplies in most tissues of adult Drosophila melanogaster, causing marked pathogenic effects and sometimes death (Chtarbanova et al. 2014).

There is considerable genetic variation in susceptibility to both of these viruses within natural populations of $D$. melanogaster (Magwire et al. 2012). Much of this variation is caused by major-effect polymorphisms that confer a high level of resistance. In the case of DMelSV, three polymorphic resistance genes have been identified: p62 [ref(2)P] (Contamine et al. 1989; Bangham et al. 2008), CHKov1 (Magwire et al. 2011), and Ge-1 (Cao et al. 2016). In a North American population, p62 and CHKov1 together explain $37 \%$ of the genetic variance in susceptibility to DMelSV (Magwire et al. 2011). Resistance to DCV is controlled by a very small number of genes, with a SNP in a gene called pastrel (pst) on chromosome 3 explaining $47 \%$ of the genetic variance in DCV susceptibility (Magwire et al. 2012). In another mapping population of flies, we recently reported that this gene accounted for $78 \%$ of the genetic variance (Cogni et al. 2016).

Despite its key role in virus resistance, pst remains poorly characterized. Its molecular function remains unknown, although it has been reported to participate in olfactory learning (Dubnau et al. 2003), protein secretion (Bard et al. 2006), and to be associated with lipid droplets (Beller et al. 2006). We identified the gene using an association study on 185 lines from North America with complete genome sequences (Mackay et al. 2012). In this study, six SNPs were found to be associated with resistance to DCV at $P<10^{-12}$, including two adjacent SNPs in the 3' UTR (T2911C and A2912C), two nonsynonymous SNPs (G484A and A2469G), and two SNPs in introns (C398A and A1870G). All of these are in linkage disequilibrium (LD), and the nonsynonymous SNP A2469G in the last coding exon stands out as the most significant polymorphism (Magwire et al. 2012). However, the strong LD between SNPs prevents us from identifying the causal SNP(s).

In this study, we have characterized genetic variation in pst and its effects on susceptibility to viral infection. In a sample of seven copies of the gene from natural populations, we find four functionally distinct alleles that confer varying levels of resistance. By combining association studies and transgenic techniques, we identify an amino acid substitution that has led to a large increase in resistance. This appears to be a relatively old polymorphism that has been maintained at a relatively stable frequency in natural populations. The pst locus also contains complex structural variation and cis-regulatory variation affecting gene expression. Higher levels of pst expression are associated with increased resistance. Therefore, this is a complex gene in which multiple genetic variants affecting both gene expression and the amino acid sequence alter susceptibility to viral infection.

\section{Materials and Methods}

\section{Generating transgenic flies carrying alleles of pst modified by recombineering}

To test which SNPs in pst are causing flies to be resistant, we used recombineering to modify a bacterial artificial chromosome (BAC) clone of the region of the Drosophila genome containing the gene (Warming et al. 2005). This allowed us to make precise modifications of six candidate SNPs previously identified in pst, with five BACs carrying each SNP separately [SNP T2911C and SNP A2912C are adjacent and in complete LD in nature, so were considered as single locus TA2911(2)CC].

Drosophila P[acman] BACs were obtained from the BACPAC Resources Center (Venken et al. 2006, 2009). The CHORI-322$21 P 14$ clone, which covers a region of the fly genome that includes pst (genome positions: 3R: 2,114,276-21,164,956), was chosen for its smaller size $(20.064 \mathrm{~kb})$ and therefore higher transformation efficiency (Venken et al. 2009). This BAC does not contain any duplication or deletion of pst.

In the BAC clone containing pst, we modified the candidate SNPs controlling resistance using recombineering and GalK positive-negative selection following Warming's protocol (Warming et al. 2005). GalK-targeting cassettes were PCR amplified from vector pgalK (Warming et al. 2005) using five different pairs of primers, each of which has $\sim 80 \mathrm{bp}$ of sequence homologous to pst at each $5^{\prime}$ end. DNA fragments of size ranging from $300 \mathrm{bp}$ to $1 \mathrm{~kb}$ that contained the SNP of interest were amplified using three Drosophila Genetic Reference Panel (DGRP) lines (Mackay et al. 2012) as template. Phusion High Fidelity polymerase (NEB) was used in the following conditions: $95^{\circ}$ for $4 \mathrm{~min}$; then $95^{\circ}$ for $15 \mathrm{sec}, 55^{\circ}$ for $30 \mathrm{sec}$, and $68^{\circ}$ for $1 \mathrm{~min}$ ( $1 \mathrm{~min}$ per $1 \mathrm{~kb}$ product), for 35 cycles; and incubate at $68^{\circ}$ for $5 \mathrm{~min}$. PCR products were gel purified using Invitrogen (Carlsbad, CA) PureLink Quick Gel Extraction Kit and fresh products were always used for transfection. Fly stocks used as template and primers used in PCR were listed in Supplemental Material, Table S1 in File S1.

We next inserted the five modified BAC clones containing the different pst alleles into identical sites in the genome of a fly line. This was possible as the BACs contain an $a t t B$ site, which allows them to be inserted into attP docking sites of flies (Bischof et al. 2007). Plasmids of concentration between 0.1 and $0.3 \mu \mathrm{g} / \mu \mathrm{l}$ and an $\mathrm{OD}_{260} / \mathrm{OD}_{280}$ ratio between 1.8 and 1.9 were injected into the embryos of an attP line: $\left.y^{-} w^{-} M^{(e G F P, ~ v a s-i n t, ~ d m R F P ~}\right) Z H-$ $2 A ; P\{$ Cary $P\}$ attp40. Injected flies were crossed to a balanced pst hypomorphic mutant to generate line $w$;transgenic; $P^{G S V 1}$ GS3006/TM3,Sb ${ }^{1} \mathrm{Ser}^{1}$. This balanced hypomorphic mutant has a $P$ element inserted in the $5^{\prime}$ UTR of the pst gene and has a lower pst messenger RNA expression level than many laboratory fly stocks we tested. The fly crossing scheme is shown in Figure S1 in File S1. 


\section{Overexpressing pst in flies}

Transgenic flies that overexpress two different $p s t$ alleles were generated using vector $\mathrm{pCaSpeR}$-hs fused with $p s t$ sequence. Expression of pst was under the control of the HSP70 promoter, and the protein is tagged by the FLAG epitope in the $\mathrm{N}$ terminus. The two pst alleles were amplified from complementary DNA from the fly lines DGRP-101 and DGRP-45 (Bloomington Drosophila Stock Center). These two DGRP lines encode identical pst amino acid sequences except for the Ala/Thr difference caused by SNP A2469G. Plasmids carrying different pst alleles were injected into a docker fly line containing an attP site on the

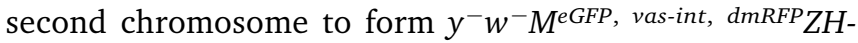
$2 A ; P\{$ CaryP $\}$ attp 40 . The experiment was subsequently repeated with a different fly line with a different attP site: $y^{-} w^{-} M^{e G F P}$, vas-int, dmRFP $Z H-2 A ; M^{a t t P} Z H-86 F b$. Male adults were crossed to the white-eye balancer $w^{1118 i s o} / y^{+} Y$;Sco/ $S M 6 a ; 3^{i s o}$ to select for successful transformants. Male and female transformants were crossed to generate homozygotes. The attP docker $y^{-} w^{-} M$ eGFP, vas-int, dmRFP $Z H$ $2 A ; P\{$ CaryP $\} a t t p 4 O$ and the balancer used in crosses $w^{1118 i s o} / y^{+} Y$;Sco/SM6a; $3^{\text {iso }}$ were used as controls for measuring DCV mortality and viral titer. Two replicates (A and B) for each of the two pst alleles were established from independent transformation events. Western blot with FLAG antibody were carried out using adult flies that were kept in $25^{\circ}$ to confirm the expression of FLAG-tagged pst alleles.

To assay the susceptibility of these lines to $p s t$, vials were set up containing 10 females and 10 males of the transgenic lines and kept in $25^{\circ}$. The parental flies were removed and the progeny collected. A total of 15 vials containing 203 - to 5-days-old mated females of each line were inoculated with DCV (or Ringer's solution as a control) as describe below, and their mortality was monitored for 19 days. Meanwhile, 15 additional vials of each line with 15 mated females were inoculated with DCV and maintained at $25^{\circ}$. At day two postinfection, total RNA of these flies was extracted and used to measure viral RNA levels by quantitative real-time PCR (qRT-PCR) (see below).

\section{Measuring pst expression in DGRP lines}

To study natural variation in gene expression, we measured $p s t$ expression in a panel of inbred fly lines from North America called the DGRP lines. We assayed 196 fly lines using one to seven biological replicates (a total of 654 RNA extractions). The flies were aged 6-9 days and a mean of 15 flies was used for each RNA extraction. These RNA extractions had been generated as part of a different experiment and were infected with Nora virus [pst is not associated with susceptibility to Nora virus (R. Cogni, personal communication) and expression of pst is not affected by Nora virus infection (Cordes et al. 2013)]. Primers and probes used are described below.

\section{Genotyping and naming of SNPS}

DNA was extracted using either DNeasy Blood and Tissue Kit (QIAGEN, Valencia, CA) according to the manufacturer's protocols or using a Chelex extraction which involved digesting fly tissues for $1 \mathrm{hr}$ at $56^{\circ}$ with 5\% w/v Chelex 100 Ion Exchange Resin (Bio-Rad, Hercules, CA) in $200 \mu$ l of $33 \mathrm{mM}$ dithiothreitol with $20 \mu g$ proteinase K (Jiggins and Tinsley 2005).

Diagnostic primers were designed to amplify the pst allele carrying specific SNPs. The SNP of interest was put at the 3' end of one primer and at least one mismatch next to the SNP was introduced (Table S2 in File S1). To experimentally confirm the structural variants of $p s t$, primers were designed to overlap the breakpoints of duplications and deletions (Table S2 in File S1). PCR products were run on $1 \% \mathrm{w} / \mathrm{v}$ agarose gels.

SNPs were named according to their position in the pst gene. Numbering begins at the nucleotide encoding the start of the 5' UTR, and includes intronic positions. The numbering of duplications and deletions refers to the size of the region affected in nucleotides. In the text, we also report the genome coordinates of all variants.

\section{DCV}

DCV stain C (Jousset et al. 1972) was kindly provided by Luis Teixeira (Teixeira et al. 2008) and was cultured in D. melanogaster DL2 cells using the protocol described in Longdon et al. (2013). The tissue culture infective dose 50 (TCID50) was calculated by the Reed-Muench end-point method (Reed 1938).

\section{Infection and resistance assay}

Newly emerged flies were tipped into new food bottles. After 2 days, mated females were infected with DCV by inoculating them with a needle dipped in DCV suspension as described in Longdon et al. (2013) $\left(\right.$ TCID50 $\left.=10^{6}\right)$. Infected flies were kept on cornmeal food without live yeast on the surface. Numbers of infected flies that died were recorded every day and surviving flies were tipped onto new food every 3 days. Flies that died within $24 \mathrm{hr}$ were excluded from the analysis as it was assumed that they died from the injection process. Infected flies were collected on day two postinfection for the measurement of viral titers.

\section{Quantitative real-time PCR}

RNA was extracted using TRIzol (Invitrogen) in a chloroformisopropanol extraction following the manufacturer's instructions. RNA was used as template in qRT-PCR using QuantiTect Virus + ROX Vial Kit (QIAGEN). Dual-labeled probes and primers were ordered from Sigma-Aldrich. The PCR primers and probes amplifying both the reference gene and the gene of interest were multiplexed in a single PCR reaction. DCV titer was measured using probe DCV_TM_Probe ([6FAM $] 5^{\prime}$ CACAACCGCTTCCACATATCCTG-3' [BHQ1]) and primers DCV_qPCR_599_F (5'-GACACTGCCTTTGATTAG-3') and DCV_qPCR_733_R (5'-CCCTCTGGGAACTAAATG-3'). The amount of virus was standardized to a reference gene RPL32 using probe Dmel_RpL32_TM_Probe ([HEX]ACAA CAGAGTGCGTCGCCGCTTCAAGG[BHQ1]) and primers Dmel_RpL32_F (5'-TGCTAAGCTGTCGCACAAATGG-3') and Dmel_RpL32_R (5'-TGCGCTTGTTCGATCCGTAAC-3'). Expression of pst was measured using dual-labeled probe 
Pst PR ([Cy5]CAGCACACCATTGGCAACTC [BHQ3]) and primers Pst_FW (5'-CCGTCTTTTGCTTTCAATA-3') and Pst_RV (5'-CCCAACTGACTGTGAATA- ${ }^{\prime}$ ). The amount of pst expression was standardized to a reference gene Ef1alpha100E using the $\Delta \Delta \mathrm{Ct}$ (critical threshold) method (see below). Expression of Ef1alpha100E was measured using probe ([FAM] CATCGGAACCGTACCAGTAGGT [BHQ2]), primers Ef1alpha100E_FW (5'-ACGTCTACAAGATCGGAG-3') and Ef1alpha100E_RV (5'-CAGACTTTACTTCGGTGAC-3'). Subsequent to the experiment, we realized there was a SNP segregating in the sequence to which the probe Pst_PR annealed, so the effect of this was corrected for by estimating the effect of this by linear regression and correcting the $\Delta \mathrm{Ct}$ values for its effect. This procedure did not qualitatively affect the conclusions. The estimation of gene expression or viral titer assumed that that the PCR reactions were $100 \%$ efficient. To check whether this assumption is realistic we used a dilution series to calculate the PCR efficiency. Three technical replicates of each PCR were performed and the mean of these was used in subsequent analyses. All the PCR efficiencies were between 97 and 103\%.

\section{Statistical analysis of survival data and viral titers}

R version 3.2.1 (R Development Core Team 2011) was used for statistical analyses. In the experiments using flies overexpressing pst and/or flies transformed with a modified BAC clone, we recorded the life span of individual flies. These data were analyzed with a Cox proportional hazard mixed model, fitted using the R package "coxme." The genotype of the fly line was treated as a fixed effect. The random effects were the vial in which a fly was kept, which was nested in the replicate fly line (where the same fly genotype had been generated twice by independent transformation events). Flies alive at the end of the experiment were censored.

For each fly line in which we measured viral titers by qRTPCR, we first calculated $\Delta \mathrm{Ct}$ as the difference between the cycle thresholds of the gene of interest and the endogenous controls (actin 5C or Ef1alpha100E). We used the mean values of technical replicates. To assess whether these differences were statistically significant, we fitted a general linear mixed model using the lme function in $\mathrm{R}$. We used the mean $\Delta \mathrm{Ct}$ across all biological replicates as a response variable. The genotype of the fly line was treated as a fixed effect and the day that the flies were injected as a random effect.

\section{Identifying structural variants of pst}

We identified structural variants by looking at the sequence data of the DGRP genomes (Mackay et al. 2012). Structural variants were detected when two halves of the same sequence read or read pair map to different positions or orientations within the reference genome. We analyzed 205 Freeze 2 BAM files of the DGRP lines (Mackay et al. 2012) using Pindel_0.2.0 (Ye et al. 2009) to identify the breakpoints of structural variants among the lines (deletions, tandem duplications, and large and small insertions). In 178 of the 205 lines (from which fly DNA was available), we confirmed the structural variants by carrying out PCR using primers either overlapping breakpoints or flanking them (Table S2 in File S1). We repeated this twice for the small number of lines that showed conflicting results with the Pindel analysis. We also Sanger sequenced the breakpoints in a subset of lines to confirm the predictions from the short-read analysis.

Duplications and deletions can also be detected by changes in sequence coverage. A script written in Python was used to calculate the coverage number for each base pair in the region 3L: 7,338,816-7,366,778 (BDGP 5).

\section{Identifying multiple alleles of pst with different effects on DCV susceptibility}

We have previously measured survival after DCV infection in a panel of inbred fly lines called the Drosophila Synthetic Population Resource (DSPR) panel B (King et al. 2012a,b). These lines were constructed by allowing eight inbred founder lines with complete genome sequences to interbreed for 50 generations, and then constructing recombinant inbred lines (RILs) whose genomes were a fine-scale mosaic of these founders. We infected 619 RILs in panel B with DCV and monitored the mortality of 14,091 flies postinfection, which allowed us to identify pst as a major-effect gene defending flies against DCV infection (Cogni et al. 2016).

In this study, we reanalyzed this data set to test whether there were more than two alleles of pst. To identify different alleles of pst, we used a hidden Markov model (King et al. 2012a) to determine from which of the eight founder lines the pst allele had been inherited. We assigned RILs to one of the founders when position 3L: 7,350,000 (the location of pst) could be assigned to that parent with $\geq 95 \%$ confidence. We analyzed this data with a one-way ANOVA, with the mean survival time of each vial RIL as the response variable, and founder allele as a fixed effect. We then performed a Tukey's honest significant difference test to assign the founders into allelic classes with differing levels of resistance.

\section{Identifying cis-regulatory polymorphisms in pst}

To look for cis-regulatory polymorphisms that cause variation in pst expression, we used a set of microarray data of female head tissue in the DSPR (King et al. 2014). The mean normalized expression of three pst probes that did not contain any SNPs segregating in the panel (FBtr0273398P00800, FBtr0273398P01433, and FBtr0273398P01911) were used. The QTL analysis was performed using the R package DSPRqtl (http://FlyRILs.org/Tools/Tutorial) (King et al. 2012b) following Cogni et al. (2016).

\section{Association between pst expression and DCV resistance}

To test whether the structural variants were associated with $p s t$ expression or susceptibility to DCV, we genotyped 178 DGRP lines for structural variants by PCR (primers listed in Table S2 in File S1). These variants were then combined with sequence data from the DGRP lines (Freeze 2). We have previously measured the survival of these fly lines after DCV infection (Magwire et al. 2012). The mean pst expression level was measured in 196 DGRP lines (see above). We then tested 
for associations between the SNPs in the region of 3L: 7,311,903-7,381,508 (BDGP5) and the mean of pst expression of each DGRP line using a linear model.

To estimate the genetic correlation between pst expression and survival after DCV infection in the DGRP lines, we used a bivariate general linear mixed model. The mean survival time of flies post-DCV infection was calculated for each vial assayed. Pst expression was measured on whole vials of flies. pst expression and survival were expressed as Gaussian response variables in the model:

$$
y_{k, i, j}=t_{k}+b_{k, i}+\varepsilon_{k, i, j},
$$

where $y_{i, j, k}$ is the observed trait $k$ (pst expression level or mean survival time) of flies from line $i$ in vial $j . t_{k}$ is a fixed effect representing the mean expression level $(\Delta \mathrm{Ct})$ or survival time. $b_{k i}$ are the random effects, which are assumed to be multivariate normal with a zero mean. For the random effects we estimated a two-by-two covariance matrix describing the genetic (between-line) variances of pst expression and survival, and the covariance between these traits. The genetic correlation was calculated from these parameters. $\varepsilon_{k ; i ; j}$ is the residual error, with separate residual variances estimated for the two traits. The parameters of the models were estimated using the R library MCMCglmm (Hadfield 2010), which uses Bayesian Markov chain Monte Carlo techniques. Each model was run for 1.3 million steps with a burn-in of 300,000 and a thinning interval of 100 . Credible intervals on all parameters (variances, correlations, etc.) were calculated from highest posterior density intervals. The analysis was repeated including SNP A2469G as a fixed effect to control for any confounding effects of this variant being in $\mathrm{LD}$ with cis-regulatory polymorphisms (assuming this SNP is not itself a cis-regulatory polymorphism).

\section{Test for natural selection on pst}

To investigate the frequency of resistance allele of A2469G in populations worldwide, we looked at publically available genome resequencing data sets of the Global Diversity Lines (Grenier et al. 2015), North American population (DGRP) (Mackay et al. 2012), and Zambian population [Drosophila Population Genomics Project (DPGP)] (Pool et al. 2012). We also collected 341 iso-female D. melanogaster from Accra, Ghana and genotyped a pool of flies from these lines for SNP A2469G by PCR as described above.

To test for a signature of natural selection on pst, we analyzed the sequence around pst from publically available genome sequences of Drosophila. These sequences were either from inbred lines or haploid genomes, so the data were phased as haplotypes. We analyzed data from two populations of $D$. melanogaster with large sample sizes: a North American population (DGRP, 205 lines) and a Zambian population (DPGP3, 197 lines). The variant calls from these lines in VCF file format of Freeze 2 DGRP was downloaded from the Baylor College of Medicine, Human Genome Sequencing Center Web site (Mackay et al. 2012). Because duplication and rearrangement of $p s t$ is very common in $D$. melanogaster, in the DGRP lines we Sanger sequenced pst from 35 lines of variant 3 and 28 lines of variant 4 so that we only analyzed data from the complete copy of the gene. These sequences were combined with 105 DGRP lines without rearrangement, resulting in a total of 165 DGRP lines with pst sequences. This was not possible for the data from Zambia as the original lines are not available. Here, consensus sequences of 197 D. melanogaster samples were downloaded from http://www.dpgp.org/. About 20-kb sequence around pst (3L: 7,340,375-7,363,363) were pulled out from all lines using the scripts "breaker.pl" and "dataslice.pl" written by the authors, returning FastA files. Then FastA file was converted into a VCF file by PGDSpider (Lischer and Excoffier 2012).

To examine how allele frequencies differ between populations, $F_{\mathrm{ST}}$ was calculated on a per-site basis for a North American population (DGRP) and a Zambian population (DPGP3) by VCFtools (Danecek et al. 2011). To detect LD around SNP A2469G, we estimated LD between all pairs of SNPs in a 20-kb region around it. The R packages "genetics" and "LDheatmap" (Shin et al. 2006) were used to calculate and plot LD in a heat map. We then applied the long-range haplotype test (Sabeti et al. 2005; Zeng et al. 2007) to examine the extended haplotype homozygosity (EHH) around SNP A2469G in comparison with other haplotypes of similar frequency in the $200-\mathrm{kb}$ region (3L: 7,250,375-7,253,363). The $\mathrm{R}$ package "rehh" was used in the analysis (Gautier and Vitalis 2012).

We finally applied a McDonald and Kreitman test (MKT) to detect positive selection on the amino acid level (McDonald and Kreitman 1991). Using the D. yakuba sequence as an outgroup, substitutions were polarized along the lineage leading from the common ancestor of D. melanogaster and D. simulans to D. melanogaster. A standard MKT was carried out using MKT software (Egea et al. 2008). We excluded polymorphic sites with a frequency of $<10 \%$ to reduce the number of deleterious amino acid polymorphisms in the data set. Polarized two-by-two contingency tables were used to calculate $\alpha$, which is an estimate of the proportion of amino acid substitutions fixed by selection (Smith and Eyre-Walker 2002). Statistical significance of the two-by-two contingency tables was determined using a $\chi^{2}$ test.

Nucleotide diversity was calculated using DnaSP version 5 (Librado and Rozas 2009) for the 20-kb region described above in 165 DGRP lines and 197 DPGP lines.

\section{Data availability}

For fly stocks and primers see Table S1 and Table S2 in File S1. The raw data and scripts used in this study are available in the University of Cambridge data repository at http://dx.doi.org/ 10.17863/CAM.866.

\section{Results}

\section{The pst locus has multiple alleles affecting DCV resistance}

In a previous association study we found six SNPs in pst that were strongly associated with DCV resistance (Magwire et al. 
2012). All of these are in LD with each other, so it was not possible to identify the causative variant from these data. Intriguingly, however, no single SNP could explain all the effects of pst on DCV susceptibility, suggesting that multiple alleles of this gene with different susceptibilities might be segregating in populations. To investigate this further, we reanalyzed a second data set where we had infected 13,919 flies from the DSPR [panel B, 619 RILs founded by eight lines representing a worldwide sample (King et al. 2012b)] with DCV and shown that resistance was largely controlled by pst (Cogni et al. 2016). These data allow us to estimate the effect that each of the seven different founder haplotypes of pst segregating among these lines has on DCV susceptibility (one of the eight founders, BB5, was removed from analysis because it is represented by $<10$ lines and was not able to be assigned to any group). The seven founder haplotypes fall into four groups with significantly different resistance levels (Figure 1). Flies in the resistant 1 (resist1) group survived an average of 9.6 days postinfection while flies in the resistant 2 (resist2) group survived an average of 11 days postinfection. Flies in the susceptible 1 (susc1) group survived an average of 6.1 days postinfection while flies in the susceptible 2 (susc2) group survived an average of 7.1 days postinfection (Figure 1). Therefore, in a sample of seven copies of this gene, there are four functionally distinct alleles of pst affecting DCV resistance.

\section{The amino acid substitution A2469G can explain resistance in two different genetic mapping experiments}

We examined the six pst SNPs previously found to be associated with resistance in our genome-wide association study in DGRP lines $\left(P<10^{-12}\right)$ and asked which of them explain the four levels of resistance we observed in the DSPR founders. Only A2469G, which is a nonsynonymous change (Thr/Ala, 3L: $7,350,895$, BDGP5), can explain the large difference between the two resistant and the two susceptible classes of alleles (Figure 1). This change is also the most significant SNP in the association study using the DGRP lines (Magwire et al. 2012) and in a separate study that had selected populations for DCV resistance and then sequenced their genomes (Martins et al. 2014). This threonine to alanine change is a radical substitution between a polar and a nonpolar amino acid, and alanine is associated with increased resistance in both the association study and this QTL analysis. Two closely related species, D. simulans and D. yakuba, both have a threonine at this position; indicating that the susceptible allele was the ancestral state. While this analysis strongly implicates A2469G in resistance, it does not preclude a role for the other five variants associated with resistance. For example, SNP C398A differs between the susc1 and susc2 alleles, while SNPs TA2911(2)CC, A1870G, and C398A all differ between resist1 and resist2.

\section{Modifying SNP A2469G in transgenic flies confirms that it alters resistance to $D C V$}

To experimentally confirm the SNP(s) causing flies to be resistant to $\mathrm{DCV}$, we generated five transgenic lines where we modified each of the six SNPs associated with resistance [SNP T2911C and A2912C, which are in complete LD, were modified together: TA2911(2)CC]. To do this we edited a BAC clone (CHORI-322-21P14, $20.064 \mathrm{~kb}$ ) of the region in Escherichia coli. The BAC originally contains the allele associated with increased resistance for all five of the pst variants, and we individually changed these to the susceptible variant. We inserted the five BACs into the same genomic position in a fly line to generate five transgenic lines. We crossed these transgenic flies to a balanced pst hypomorphic mutant $y^{1} w^{67 c 23}$; If/Cyo; $P^{G S V 1}$ GS3006/TM3,Sb ${ }^{1}$ Ser $^{1}$, which has a transposable element inserted in the $5^{\prime}$ UTR of the pst gene. The transgenic alleles did not complement the lethal effect of this mutation upstream of pst, so we infected flies that were homozygous for the transgenic pst allele on chromosome 2 and had one hypomorphic mutant allele over a balancer chromosome on chromosome 3 (pst hypomorphic mutant allele and the balancer carries the susceptible form "A" for SNP A2469G).

The two independent fly lines carrying an A for SNP A2469G, which were generated through independent transformation events, died significantly faster after DCV infection compared with all the other transgenic lines that had a "G" at this position (Figure 2). There were no significant differences among the other four genotypes. Among the flies that were mock infected with Ringer's solution, there were no significant differences among lines (although flies carrying a susceptible A at A2469G survived longest, which reinforces the result that the high mortality of these flies when DCV infected is being caused by pst). In summary, both genetic mapping approaches and experimentally modifying the gene demonstrate that the SNP A2469G is causing flies to be resistant to DCV.

\section{Overexpressing both the resistant and susceptible alleles of pst protects flies against DCV infection}

Resistance could evolve by altering host factors that are beneficial to the virus or by increasing the efficacy of existing antiviral defenses. To distinguish between these hypotheses, we generated fly lines that overexpress either the resistant or the susceptible allele of pst (these constructs encode a protein that only differs at the site affected by SNP A2469G). The two FLAG-tagged constructs were inserted at the same attp 40 of the fly genome using phiC31 integrase, and we checked that the full-length protein $(\sim 77 \mathrm{kDa})$ was being expressed using a Western blot targeting the FLAG tag. Two replicates of these lines were generated and these flies were then infected with DCV. We found that overexpressing both the susceptible and the resistant alleles of pst led to significant reductions in viral titers at 2 days postinfection (Figure 3A; general linear model: pst $_{\mathrm{A}}:|z|=3.3, P=0.003$, pst $_{\mathrm{G}}:|z|=4.83, P<$ $0.001)$. There is no significant difference in viral titer between flies overexpressing the resistant pst allele $\mathrm{G}$ and flies overexpressing the susceptible pst allele $\mathrm{A}$, although the trend is in the expected direction (Figure 3A; $|z|=1.4$, $P=0.35$ ). Next, we examined survival. Overexpressing $p s t$, 


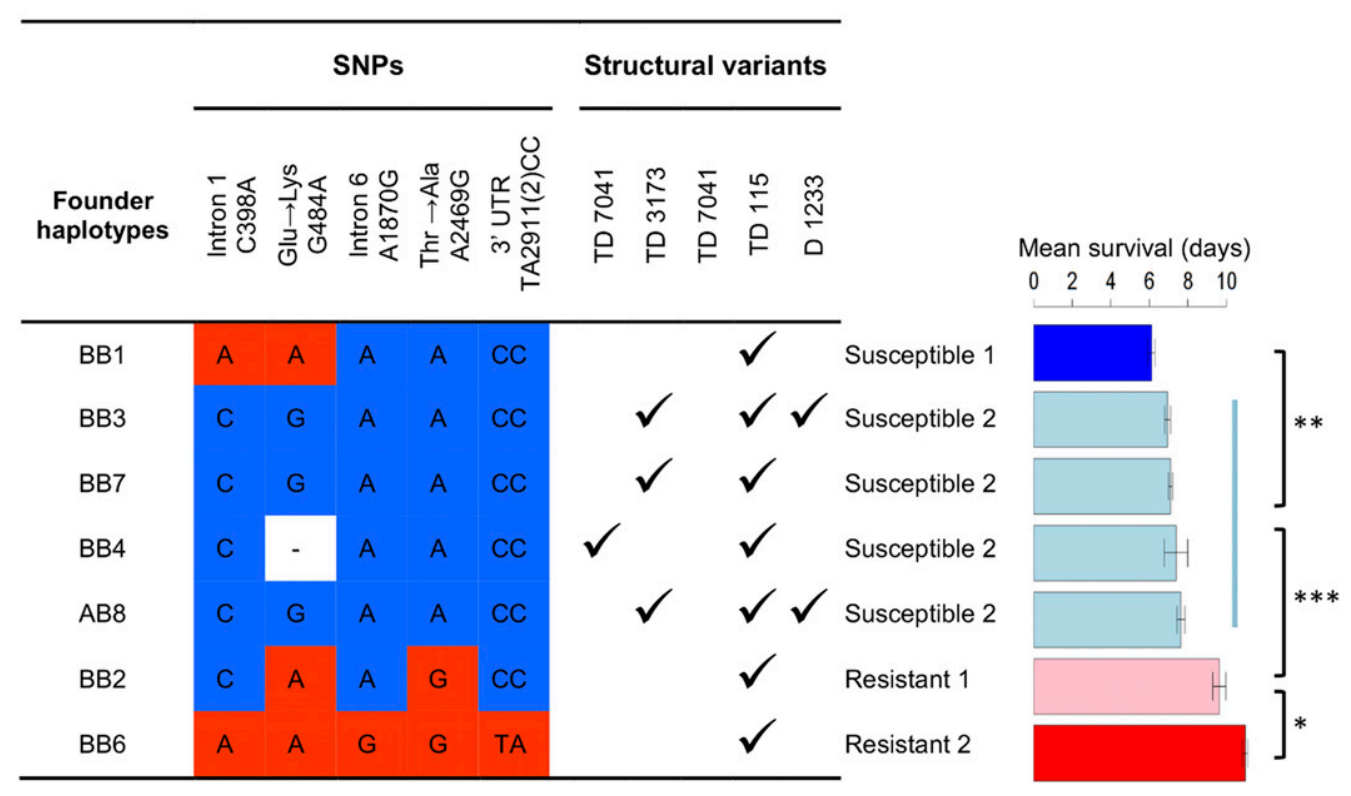

Figure 1 Genetic variation and susceptibility to DCV of the pastrel haplotypes segregating in the DSPR panel. The genotypes of SNPs that were strongly associated with resistance in a previous association study are shown (Magwire et al. 2012). Susceptible SNPs are in blue and resistant SNPs are in red. We estimated the mean survival time of the founders using an ANOVA, and identified groups of founder haplotypes with significantly different levels of resistance using Tukey's honest significant differences test. There are four phenotypically distinct classes of alleles that have significantly different effects on resistance $(* P<0.05, * * P<$ 0.01, *** $P<0.001$ ). In total, the survival of 13,919 flies was analyzed. Error bars are SEs. The locations of the SNPs in chromosome 3L of the Drosophila genome are C398A: 7352966; G484A: 7352880; A1870G: 7351494; A2469G: 7350895; TA2911(2)CC: 7350452-3. The structural variants are named according to whether they are tandem duplications (TD) or deletions (D).

no matter which allele, substantially increased survival after DCV infection (Cox proportional hazard mixed models; $\left.p s t_{A}:|z|=12.32, P<1 e^{-5}, p s t_{G}:|z|=11.83, P<1 e^{-5}\right)$ (Figure 3B). Again, we were not able to detect any difference in mortality between flies overexpressing the two different alleles of $p s t(|z|=0.53, P=0.86)$. This result should be interpreted with caution, as any differences in resistance between the two alleles may be obscured by intrinsically lower survival of the flies overexpressing the resistant allele (Figure 3B, Ringers control). This difference in the survival of mock-infected flies overexpressing the different alleles could not be replicated when new transgenic flies were generated in a different genetic background and assayed without pricking, suggesting that it is not a toxic effect of the resistant allele (Figure S2 in File S1). In summary, overexpressing either pst allele substantially increased resistance, with the resistant allele causing a slightly greater reduction in viral titer.

\section{The pst locus contains complex structural polymorphisms}

The analyses above only considered SNPs, but other types of genetic variation could cause flies to be resistant. We therefore investigated the existence of structural variation in a panel of 205 inbred fly lines from North America whose genomes had been sequenced (DGRP) (Mackay et al. 2012). The existence of structural variation had been suggested by the PCR amplification of a truncated copy of pst in certain flies and cell lines. We identified the breakpoints of structural variants from published paired-end, short-read sequencing data (using Pindel_0.2.0) (Ye et al. 2009). Excluding small indels, this approach revealed five variants that were shared by more than three lines and supported by at least four raw sequencing reads (Figure 4A; the region investigated, 3L: 7,346,678-7,357,466, DPGP 5, includes pst and the two flanking genes CTCF and Sec63). In 178 of the 205 lines we confirmed the structural variants by carrying out PCR with diagnostic primers and Sanger sequencing. As a final confirmation, we checked that the duplicated regions had increased sequence depth (Figure 4B).

The five major structural variants and their frequencies in the DGRP lines are summarized in Figure 4A. Just over half of the lines had the ancestral state which is found in the reference genome with one complete copy of pst (Figure 4A; ancestral allele). A total of 8 out of 205 lines have a 7960-bp duplication (3L: 7,348,816-7,356,777, variant 1) containing a complete copy of pst and some sequences from two adjacent genes (CTCF and Sec63). A total of 20 lines have a 7041-bp duplication (includes pst and partial sequences from neighbor genes, 3L: $7,348,778-7,355,820$, variant 2). Additionally, 35 lines have a duplicated copy of pst (3L: 7,350,246-7,353,420) with a 1233-bp deletion in the middle (variant 3 ). Finally, 32 lines have a duplication of 115 bp (3L: 7,350,263-7,350,379) at the $3^{\prime}$ end of pst (variant 4). There are another five lines containing structural variants each represented by less than three lines that are not shown in Figure 4A.

We tested whether these structural variants affect survival of the DGRP lines after DCV infection and found that none of the structural variants is associated with survival post-DCV infection $\left(F_{1,165}<1.97, P>0.32\right.$; Figure $\left.4 C\right)$. This nonsignificant result may due to a lack of power. For example, one of the structural variants that has a complete copy of pst was only represented by as few as eight lines. Another possible explanation is that transcripts produced by these duplicates are nonfunctional.

\section{There is cis-acting genetic variation that alters the expression of pst}

Given that altering the expression of pst experimentally alters resistance to DCV, it is possible that natural variation in gene 


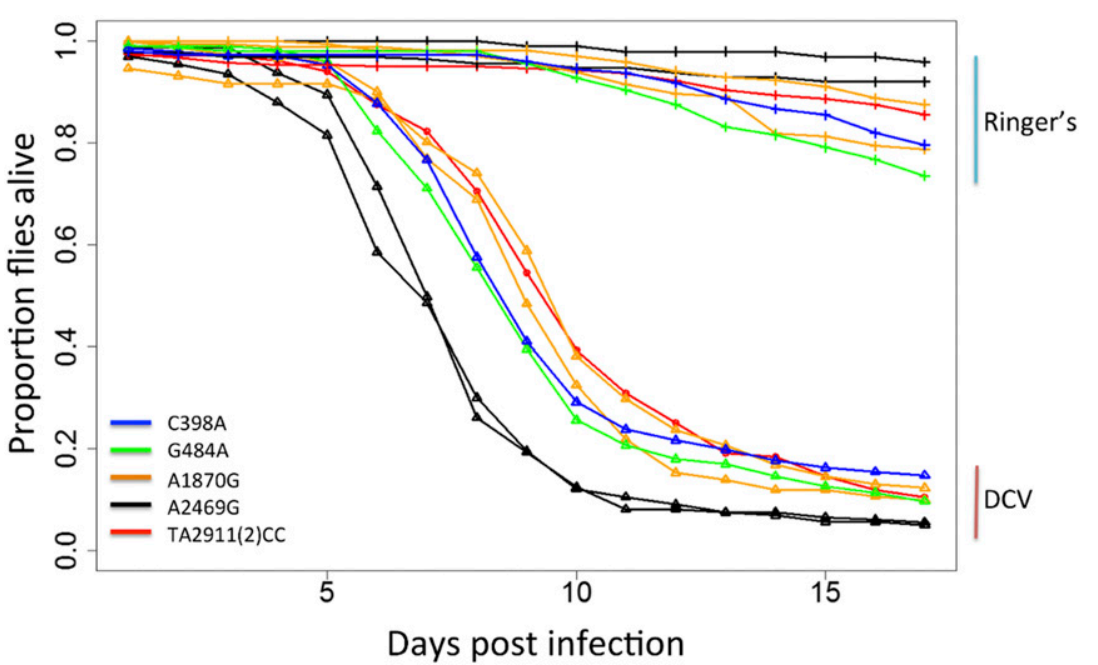

Figure 2 Susceptibility to DCV in transgenic flies carrying different alleles of pst SNPs. Lines with $\boldsymbol{\Delta}$ are flies infected with DCV while lines with + are flies injected with Ringer's solution as a control. SNP A2469G and SNP A1870G have two biological replicates, which were generated through independent transformation events. By fitting a Cox proportional hazard mixed model, we found that A2469G is significantly different from all the other SNPs $(P<0.007)$. There were no significant differences among the other four SNPS $(P>0.36)$. In total, 157 vials containing 3010 females were infected with DCV and their mortality were recorded daily for 17 days.

expression affects susceptibility to the virus. We investigated this using published microarray data from $\mathrm{F}_{1}$ individuals from crosses between two panels of recombinant fly lines derived from 15 founder lines from around the world (crosses between DSPR panel A females and panel B males) (King et al. 2014). To map regions of the genome affecting pst expression, we used the mean normalized expression of three pst probes (FBtr0273398P00800, FBtr0273398P01433, and FBtr0273398P01911) that did not contain any SNPs. We found that there was a major QTL controlling pst expression at 3L: 7,350,000 ( $\mathrm{LOD}=35.04)$, which is very close to the location of pst (Figure 5A). Therefore, there is genetic variation in pst expression and this is controlled by cis-acting genetic variants close to $p s t$ rather than variation elsewhere in the genome acting in trans.

To investigate which genetic variants might be affecting pst expression, we measured expression across 198 DGRP lines using qRT-PCR. Using these data, we looked for associations between the five structural variants and SNPs in the region surrounding pst (Figure 5B). We found pst expression was most significantly associated with a SNP in an intron of pst at position A1455T (3L: 7,351,909, $F_{2,155}=17.89, P=$ $1.02 e^{-7}$ ). However, several of the structural variants were also associated with pst expression (Figure 5B). Tandem duplications TD3173 and TD115 were in LD with SNP A1455T (Fisher's exact test: $P=0.002$ and $P=0.001$ ), but they remain significantly associated with pst expression after accounting for SNP A1455T by including it as a covariate in the model (TD3173: $F_{1,156}=14.07, P=0.0002$; $T$ D115: $F_{1,156}=$ 7.7, $P=0.006$; Figure 5B). TD3173 is in strong LD with D1233 (Fisher's Exact Test, $P<2.2 e^{-16}$ ). Therefore, multiple cis-regulatory variants affect pst expression, and these may include structural variants.

\section{The expression of pst is correlated with DCV resistance}

Across 198 DGRP lines we found that natural variation in pst expression was correlated with survival after DCV infection (Figure 6; genetic correlation: $r_{\mathrm{g}}=0.32$, 95\% C.I. $\left.=0.17-0.45\right)$. This is consistent with previous results that DCV resistance changes when pst is knocked down by RNA interference (RNAi) (Magwire et al. 2012) or overexpressed using transgenic techniques (Figure 3). SNP A2469G is a nonsynonymous SNP that was found to affect survival after DCV infection, which means it is unlikely to have an effect on gene expression. However, if it is in $\mathrm{LD}$ with a cis-regulatory variant, this could create spurious associations between gene expression and resistance. To control for this, we estimated the correlation after accounting for the effect of SNP A2469G by including it as a covariate in the model, and found the correlation between pst expression and survival after DCV infection remains significant (genetic correlation: $r_{\mathrm{g}}=0.25,95 \%$ C.I. $\left.=0.11-0.41\right)$. These results indicate that cis-regulatory variation that alters pst expression and affects resistance to DCV.

\section{There is no evidence of spatially varying selection acting on the resistant allele of pst A2469G}

Having identified the genetic variant that is responsible for most of the genetic variation in DCV resistance in $D$. melanogaster, we are well placed to characterize how natural selection has acted on this variant. It is common to find that the prevalence of viruses in Drosophila varies geographically (Carpenter et al. 2012; Webster et al. 2015), and this is expected to result in spatially varying selection pressure for resistance. However, there is little variation in the frequency of the resistant allele between populations. The resistant allele of A2469G is at a low frequency in populations worldwide: $7.7 \%$ in Zambia [197 DPGP3 lines (Pool et al. 2012)], 16\% in North America [205 DGRP lines (Mackay et al. 2012)], 10\% in Beijing [15 GDL lines (Grenier et al. 2015)], 5\% in The Netherlands (19 GDL lines), $33 \%$ in Tasmania (18 GDL lines), and 10\% in Ghana (341 lines collected and genotyped in this study). Among the populations with genome sequence data, only Zambian and North American populations have large sample sizes (197 lines and 205 lines, respectively), so the following analyses were carried out on these two data sets.

To compare the geographical variation in allele frequency at $\mathrm{A} 2469 \mathrm{G}$ to other SNPs in the region, we calculated $F_{\mathrm{ST}}$ (a measure of differences in allele frequency) between North 

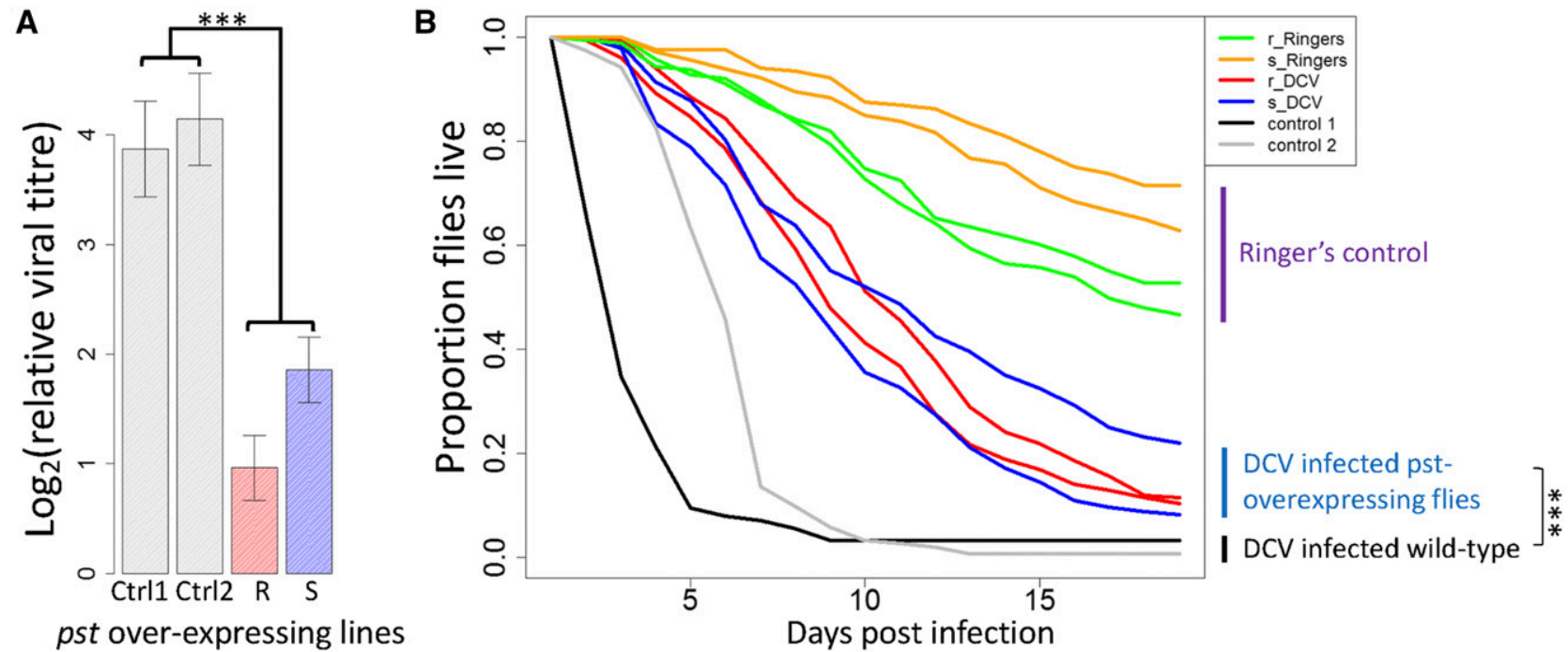

Figure 3 The effect of overexpressing pst carrying the susceptible (s) and resistant ( $r$ ) alleles of SNP A2469G on survival and viral titer. (A) DCV titer relative to $\mathrm{Act} 5 \mathrm{C}$ in flies 2 days postinfection. Bars are the means of 28 vials each containing 15 flies. Error bars are SEs. (B) The proportion of flies alive after infection with DCV or mock infection with Ringer's solution. The survival curves are the mean of $\sim 15$ vials of flies, with a mean of 18 flies in each vial. Flies were kept at $25^{\circ}$. Control 1 (Ctrl1) were docker flies into which the BAC constructs were inserted $\left(y^{-} W^{-} M e^{G F P, ~ v a s-i n t, ~ d m R F P Z H-2 A ; P ~} P\right.$ CaryP $\}$ attp40), and control 2 (Ctrl2) were flies used in the crosses to select successful transformants $\left(W^{1118 i s o} / y^{+} Y ; S c o / S M 6 a ; 3^{i s o}\right)$. The experiments used two independent transformants of each construct $(\mathrm{A}$ and $\mathrm{B}) . * * * P \ll 0.001$.

America and Zambia. It is clear that A2469G (red * in Figure $7 \mathrm{~A})$ is not a significant outlier relative to the other 2641 SNPs analyzed in the 100-kbp region on either side (SNPs that have a minor allele frequency $<5 \%$ were filtered out), indicating there is no evidence of population-specific selective pressure on SNP A2469G.

\section{The resistant allele of pst is old and shows no evidence of recent changes in frequency driven by natural selection}

When natural selection causes an unusual, rapid rise in allele frequency, there is little time for recombination to break down the haplotype carrying the selected mutation. This results in unusual long-range haplotypes and elevated LD around the variant given its population frequency. As we know the site that is likely to be a target of selection, this is a powerful way to detect the effects of selection on DCV resistance. We first measured the LD between SNP A2469G and SNPs in a 10-kb region upstream and downstream of it. In both Africa and North America we found very little $\mathrm{LD}$ between SNP A2469G and surrounding SNPs (Figure S3, A and $\mathrm{B}$, in File S1).

When the variant under selection is known, the most powerful test for such effects is the EHH test (Sabeti et al. 2005; Zeng et al. 2007). We calculated the EHH using the resistant (derived) allele of SNP A2469G as a core, and compared this to a null distribution generated from other SNPs of similar frequency that were nearby in the genome (Figure 7, B and C). In both populations, although the $\mathrm{EHH}$ around the resistant allele of A2469G is above the median, it is below the top $5 \%$. Therefore, there is no evidence of positive selection on the resistant allele of A2469G generating extended LD around this variant. We also calculated the EHH for the susceptible allele of SNP A2469G as a core, and found no extended LD around this variant (Figure S4 in File S1).

Positive and balancing selection can also affect the nucleotide diversity $(\pi)$. In a 20-kb region around pst in both North American and African populations, we did not observe elevated nucleotide diversity compared to the $\pi$ value of the whole genome (Figure S5, A and B, in File S1). We also calculated $\pi$ among chromosomes carrying the resistant or the susceptible allele of A2469G, and did not find altered patterns of diversity around pst (Figure S5, C and D, in File S1).

It is common to find components of the immune system where natural selection has driven rapid evolution of the protein sequence, which is normally interpreted as being caused by selection by pathogens (Obbard et al. 2009). To test whether this was the case for $p s t$, we tested whether other amino acid variants had been fixed in pst using the MKT (McDonald and Kreitman 1991). D. yakuba and D. simulans sequences were used to infer the sequence of the most recent common ancestor of $D$. simulans and D. melanogaster. Analyzing polymorphisms from 165 lines from the DGRP panel and divergence from the most recent common ancestor of $D$. simulans and $D$. melanogaster, we found no signature of positive selection (low frequency variants excluded; synonymous polymorphism $=7$, synonymous divergence $=13.23$, nonsynonymous polymorphism $=13$, nonsynonymous divergence $=$ 32.46, $\alpha=0.76, \chi^{2}=0.242, P=0.625$ ). Therefore, there is no evidence of positive selection on the amino acid sequence of Pastrel over the last $\sim 3$ MY. 


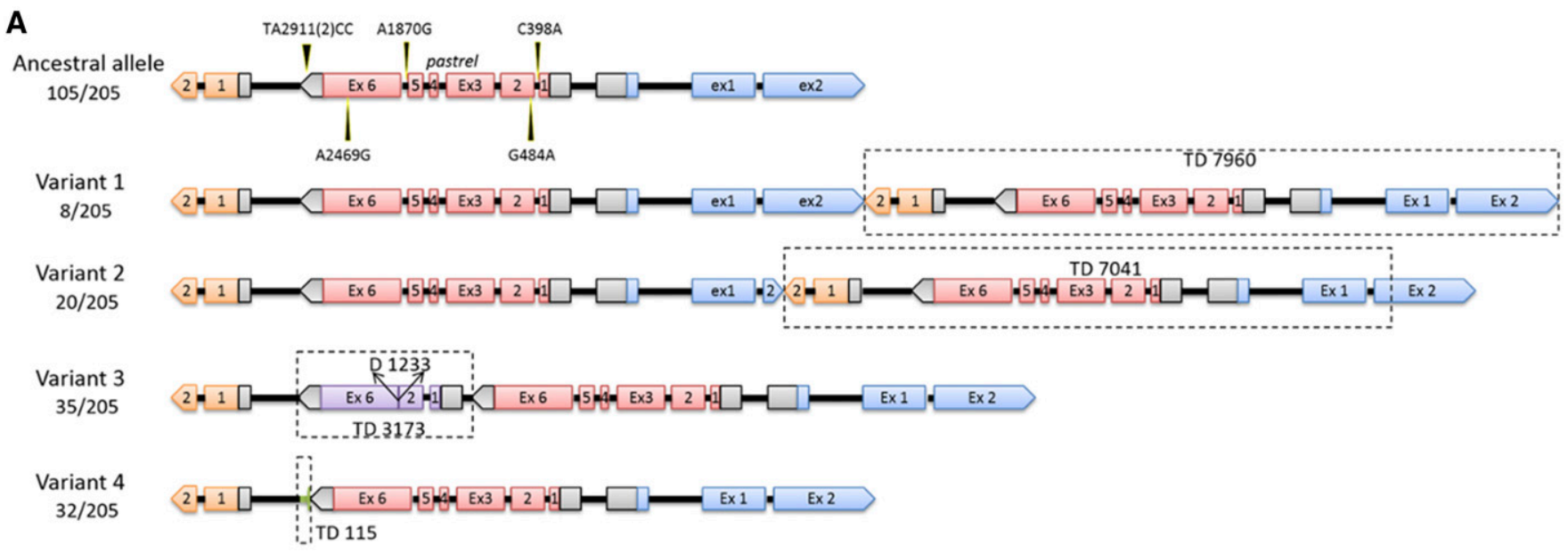

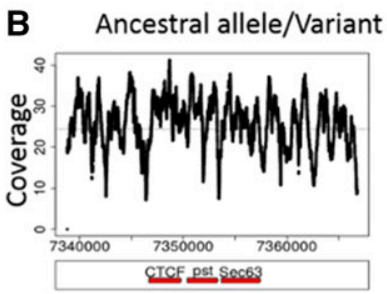

Variant 1

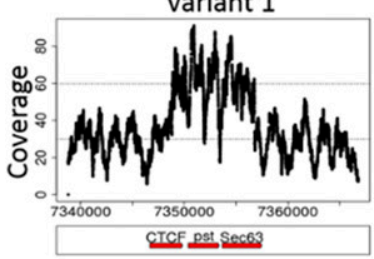

Genome position

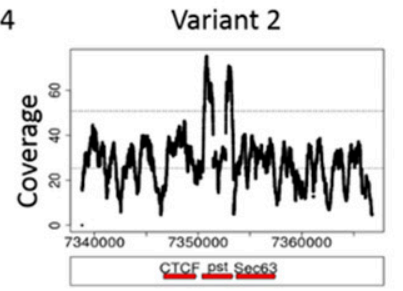

Variant 3

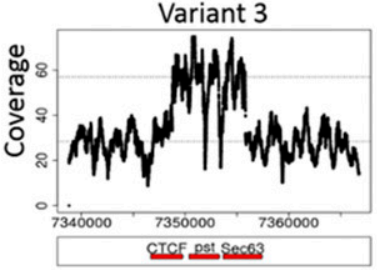

Genome position
C

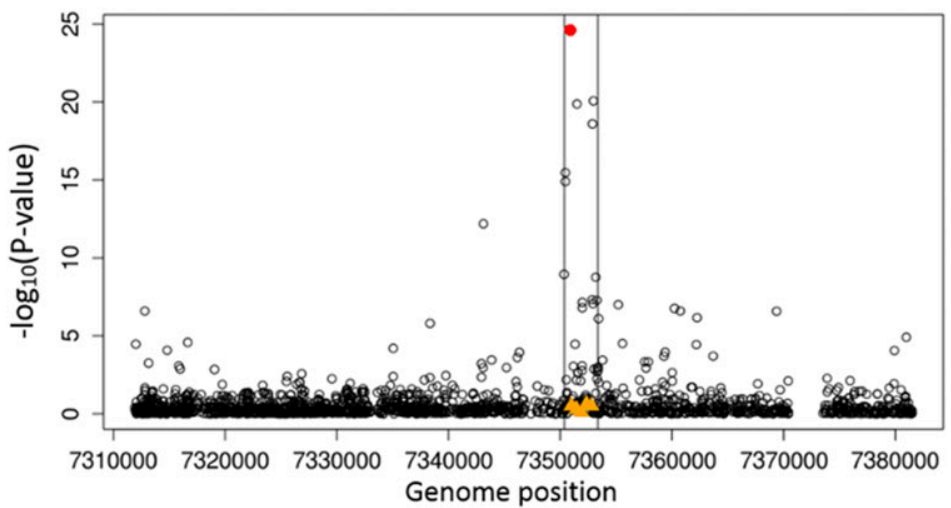

Figure 4 Five structural variants of pastrel. (A) Cartoon of pst variants, with alleles' size scaled to gene length. Pink boxes represent complete copy of pst gene; orange boxes represent coding sequence of gene CTCF located at 3' end of pst; blue boxes represent coding sequence of gene Sec63, located at 5' end of pst; gray boxes are UTRs; purple boxes are truncated copy of pst gene. Allele frequencies in DGRP are shown below the variant name. Variant 2 differs from variant 1 in that variant 2 has a shorter duplication of CTCF exon (ex) 2. (B) Mean sequencing coverage plots of the region 3L: 7,338,816 (1-kb upstream of the start of TD7960)-7,366,778 (1-kb downstream of the end of TD7960) for ancestral allele of pst and four structural variants. Red bars stand for pst and two neighbor genes CTCF and Sec63. Variant 4 has a very short duplication of 115 bp so shows very similar coverage plot as the ancestral allele. Sequence data are from the original DGRP genome sequencing project (Mackay et al. 2012). (C) Association between survival after DCV infection and pst SNPs and structural variants. $-\log _{10}(P$-value) of the association between SNPs in the region of 3L: 7,311,903-7,381,508 (BDGP 5), and survival is plotted against genome positions of the SNPs. SNPs are showed as O; SNP A2469G is in red. Structural variants of pst are showed in orange $\mathbf{\Delta}$ 's.

\section{Discussion}

It has been argued that susceptibility to infectious disease may frequently have a simpler genetic basis than many other quantitative traits because natural selection drives major-effect resistance alleles up in frequency in populations (Hill 2012; Magwire et al. 2012). At first sight susceptibility to DCV in Drosophila would appear to be a clear example of this pattern, with a restriction factor called Pastrel explaining as much as $78 \%$ of the genetic variance in this trait (Cogni et al. 2016). However, we have found that this belies considerable complexity within this locus. Strikingly, in a sample of just seven alleles from natural populations, we found four phenotypically distinct allelic classes conferring differing levels of resistance to DCV. Furthermore, both coding and cis-regulatory variants in pst control resistance. The coding sequence variant that we characterized appears to be an old polymorphism that has been maintained at a relatively stable frequency, possibly as a result of balancing selection.

As we have found for DCV, it may be common for genes affecting quantitative traits to have multiple alleles (allelic heterogeneity). The most important gene controlling resistance to the sigma virus DMelSV in D. melanogaster is CHKov1, where there are three alleles conferring differing levels of resistance (Magwire et al. 2011). Increased transcription of the detoxification gene Cyp6g1 confers resistance to insecticides in $D$. melanogaster, and again there are multiple alleles segregating in nature (Schmidt et al. 2010). One of the moststudied genes in natural populations of Drosophila is Adh, and 
A

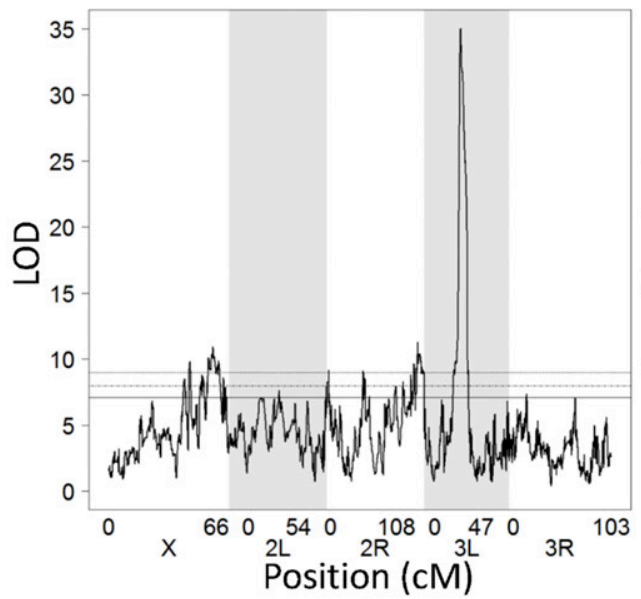

B

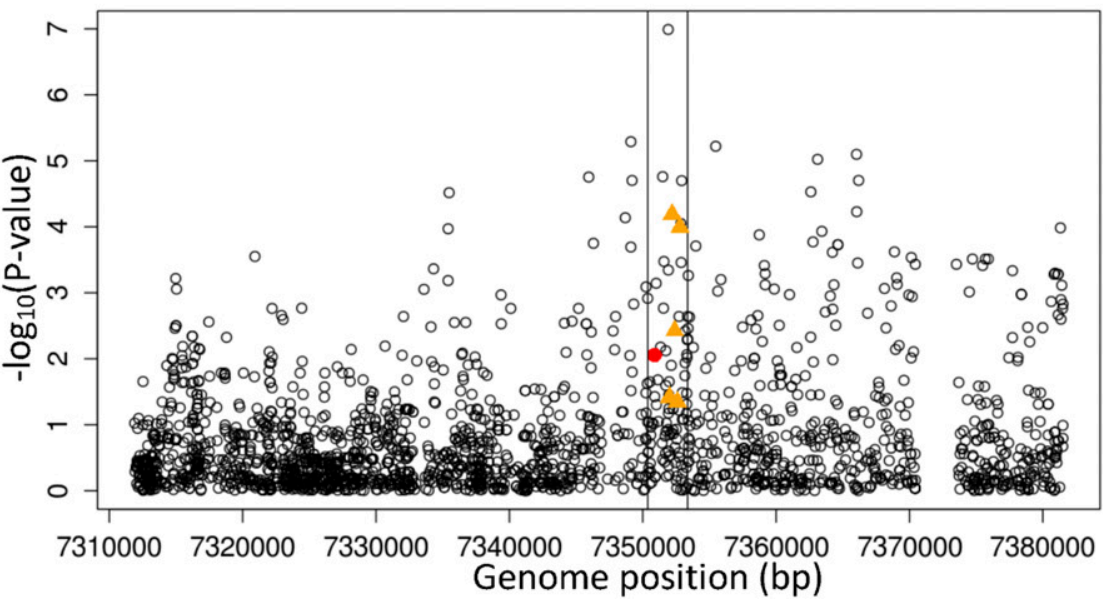

Figure 5 Cis-regulatory variation in pst expression. (A) Map of QTL associated with pst expression in female head of DSPR crosses. A single peak at position 3L: 7,350,000 was found $(\mathrm{LOD}=35)$. The horizontal line is the genome-wide significance threshold obtained by permutation $(P<0.05, \mathrm{LOD}=$ 7.12). Expression data are from published microarray analysis (King et al. 2014). (B) Association between pst expression and its SNPs and structural variants in DGRP lines. Gene expression was measured by qRT-PCR on 654 biological replicates of 196 fly lines. - Log $_{10}(P$-value $)$ for the association between SNPs and expression in the region of 3L: 7,311,903-7,381,508 (BDGP 5) is plotted against genome positions of the SNPs. SNPs are showed as $\mathrm{O}$; SNP A2469G in red. Structural variants of pst are showed in orange $\boldsymbol{\Delta}$ 's.

multiple alleles are associated with ADH activity (Laurie and Stam 1994; King et al. 2012b). Allelic heterogeneity may not be restricted to these genes with large phenotypic effects. In a recent study on gene expression in D. melanogaster, $7922 \mathrm{ex}-$ pression QTL (eQTL) were mapped, and allelic heterogeneity was found in $95 \%$ of eQTLs acting in cis and $78 \%$ of eQTLs acting in trans (King et al. 2014).

An amino acid polymorphism in Pastrel is the most important factor determining susceptibility to DCV. There are multiple lines of evidence to support this. First, this is the only genetic variant that can explain the largest changes in resistance that we see in two large genetic mapping experiments. Second, when populations have been artificially selected for DCV resistance, this site shows the largest increase in frequency in the entire genome (Martins et al. 2014). Finally, we verified the phenotypic effect of this site by modifying it in transgenic flies. These transgenic flies were generated by inserting large BACs that contained a copy of the pastrel gene along with upstream sequences. We had intended to combine this with a mutant allele of pastrel, but this cross failed, possibly because the BAC failed to complement the mutation or due to other recessive lethal alleles on the chromosome. We therefore assayed the effect of this construct in flies that had a hemizygous wild-type-susceptible allele. This may be one of the reasons why the transgenic flies did not have as large an effect on resistance as was seen in the association studies. In the future, genome editing with Cas 9 will allow the gene to be modified seamlessly in its natural location in the genome.

The ancestral state at this site was the susceptible allele threonine. Three other major-effect polymorphisms that affect susceptibility to viruses in Drosophila have been identified at the molecular level, and in all cases the ancestral state was susceptible (Bangham et al. 2008; Magwire et al. 2011; Cao et al. 2016). This fits with a model whereby genetic variation is arising because there is continual input of novel resistance alleles into populations from mutations, and these are then favored by natural selection.

Resistance could evolve by improving existing antiviral defenses or by altering the myriad of host factors hijacked by the virus for its own benefit. For example, in C. elegans, susceptibility to the Orsay virus is determined by a polymorphism that disables the antiviral RNAi defenses (Ashe et al. 2013), while bacteriophage resistance is frequently associated with changes to surface receptors used by the virus to enter cells (Longdon et al. 2014). In a previous study, we found that knocking down the susceptible allele of pst makes flies even more susceptible (Magwire et al. 2012), while in this study we found that overexpressing the susceptible allele makes flies resistant. Therefore, the threonine to alanine mutation that we observe in pst is an improvement to an existing antiviral defense.

Patterns of genetic variation at the pst locus are complex. We found extensive structural variation, with multiple duplications and deletions of the gene present in natural populations. Gene duplications and rearrangements frequently affect gene function and can be evolutionarily important. However, these variants are not annotated in the standard versions of the DGRP or DSPR panels, and it took a considerable amount of laboratory work and analysis to characterize this variation. This reflects the difficulty of identifying structural variation using short-read sequencing, and represents a limitation of these genomic resources. In this case, however, we did not find a significant association between these structural variants and resistance to DCV.

There is also genetic variation in the expression of pst. There was a single QTL that controls pst expression centered 


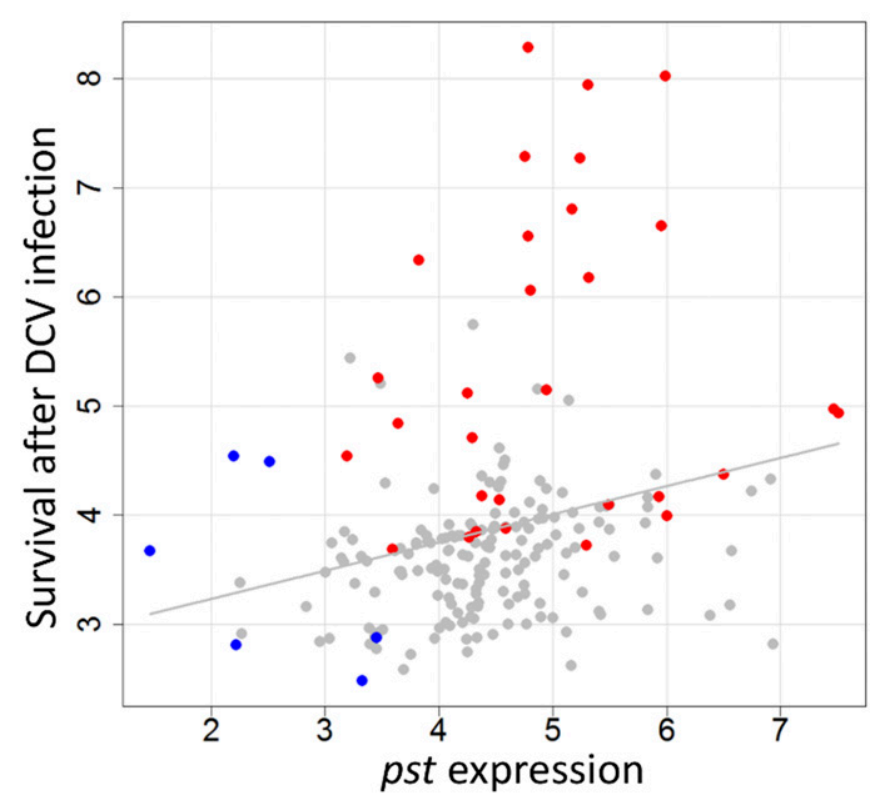

Figure 6 Correlation between pst expression and survival after DCV infection in DGRPs. Gray line is fitted by linear regression line and is shown for illustrative purposes only. Each point is the estimated phenotype of a single DGRP line (marginal posterior modes of the random effects in model Equation 1). Red $\bullet$ 's represent lines that contain resistant allele G for SNP A2469G and blue •'s represent lines contain " $T$ " for SNP A1455T. Gene expression was measured by qRT-PCR on 654 biological replicates of 196 fly lines. Survival after DCV infection was estimated from 730 vials of flies, with the data from Magwire et al. (2012).

on pst itself, suggesting that cis-regulatory variants control pst expression. Higher levels of pst expression are associated with increased resistance to DCV. This is unsurprising, as when we have experimentally altered pst expression by RNAi or by overexpressing the gene, DCV resistance is altered. Both SNPs and structural variants in the region are associated with pst expression. However, the cis-regulatory variants which are causing increased expression could not be unambiguously identified because of LD between these sites. Interestingly, the structural variants themselves were not significantly associated with survival after DCV infection, perhaps suggesting that they are not the main cause of variation in gene expression. Nonetheless, given the central role this gene plays in antiviral defense, it is tempting to speculate that these complex structural changes may have had some functional role, perhaps against other viruses (or we may simply lack the statistical power to detect effects on DCV) (Martins et al. 2014).

Why is genetic variation in susceptibility to DCV maintained in populations? There is likely to be selection favoring alleles that increase resistance in natural populations because DCV is the most virulent virus that has been isolated from Drosophila and field studies have found it to be geographically widespread (Christian 1987) [although recent surveys have suggested that it may have a low prevalence (Webster et al. 2015)]. pastrel has also been implicated in resistance to other viruses related to DCV (Martins et al. 2014). Given that the resistant allele is likely to enjoy a selective advantage, an important question is why the susceptible alleles have not been eliminated by natural selection. To understand how selection has acted on the amino acid variant that causes resistance, we examined geographical variation in its frequency and patterns of LD with neighboring sites. We could detect no evidence of natural selection causing changes in allele frequency through time or space. This is in stark contrast to the partial selective sweeps that we have seen in the two other major-effect polymorphisms affecting virus resistance (Bangham et al. 2008; Magwire et al. 2011). These polymorphisms are in the genes CHKov1 and P62 [ref(2)P] and both confer resistance to the sigma virus. In both cases the resistant allele has recently arisen by mutation and has spread through $D$. melanogaster populations under strong directional selection. In comparison to these polymorphisms it is clear that the polymorphism in pst is relatively old and does not show the same signatures of strong recent selection.

Observed population genetic patterns suggest that either the polymorphism has been evolving neutrally, or it has been maintained by balancing selection due to the benefits of resistance being balanced by harmful pleiotropic effects of the resistant allele on other traits. Long-term balancing selection can leave a signature of high divergence between the two alleles and elevated sequence polymorphism (Charlesworth 2006), but we have been unable to find any evidence of this in pst. This is not unexpected because the large effective population size of $D$. melanogaster means that $\mathrm{LD}$ declines rapidly around $p s t$, and this is expected to erode any signature of balancing selection (Charlesworth 2006). A very similar pattern of sequence variation was recently reported around a polymorphism in the antimicrobial peptide Diptericin which affects susceptibility to bacterial infection (Unckless et al. 2016). This amino acid polymorphism is also found in the sibling species $D$. simulans, strongly suggesting it is maintained by balancing selection. Therefore, we cannot distinguish balancing selection and neutral evolution. While it seems likely that a polymorphism with such a large phenotypic effect is the target of natural selection, we would need additional data from natural populations to demonstrate that this was the case.

In Drosophila, increased resistance against bacteria and parasitoid wasps is associated with reduced fecundity and larval survival (Kraaijeveld and Godfray 1997; McKean et al. 2008). However, when populations of flies were selected for DCV resistance there was no detectable decline in other components of fitness (Faria et al. 2015). Unfortunately, while it is clear the resistant allele of pst is not highly costly, this negative result is hard to interpret. First, if the benefits of DCV resistance in nature are small, then a small cost that cannot be detected in the laboratory will be sufficient to maintain the polymorphism. Without having an estimate of the harm flies suffer due to DCV infection in nature it becomes impossible to reject the hypothesis that the benefits of resistance are balanced by pleiotropic costs. Second, costs of resistance are typically only expressed in certain environments and may affect many different traits 

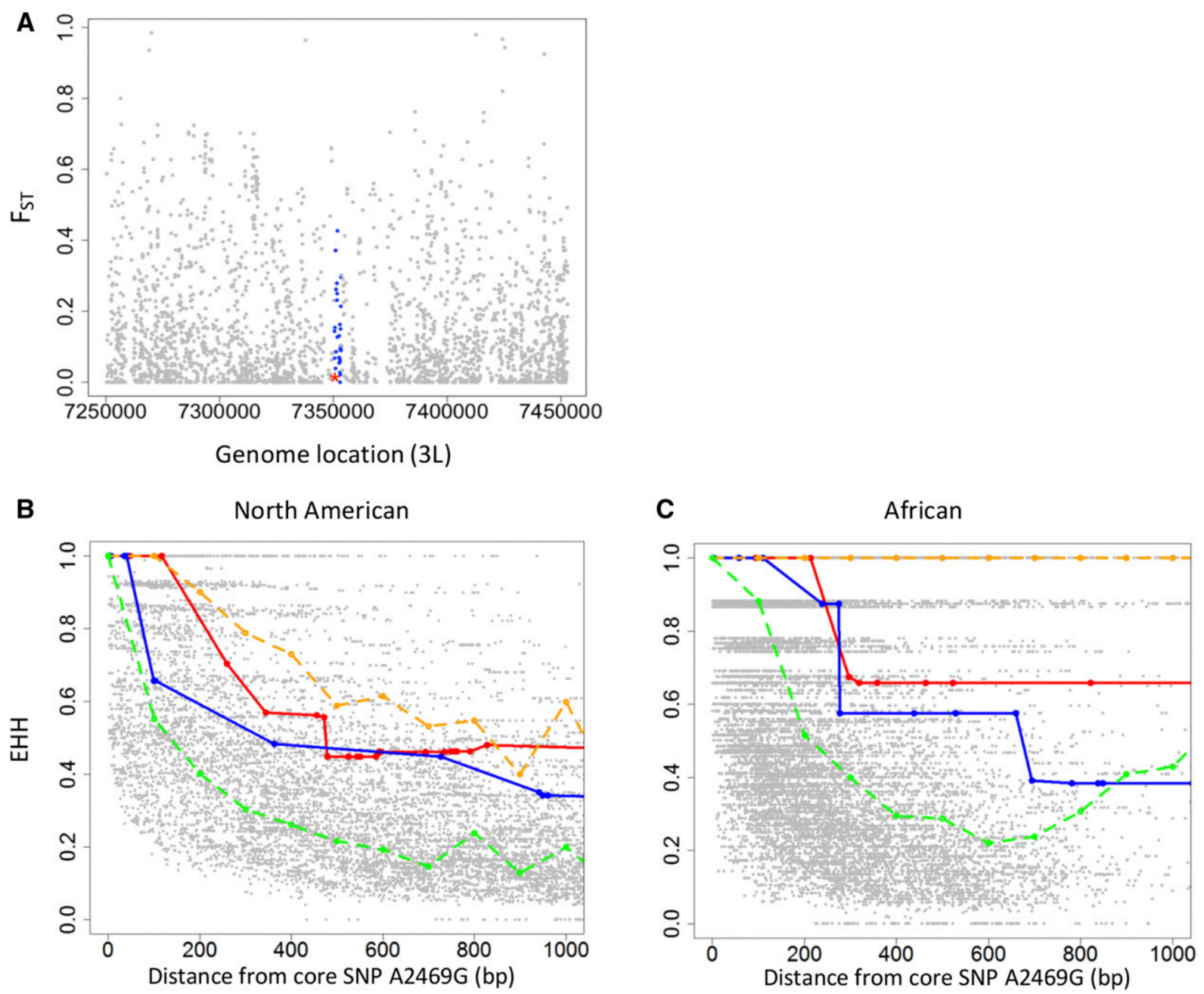

Figure 7 Population genetic analysis of natural selection acting on the amino acid polymorphism A2469G in pst that confers resistance to DCV. (A) $F_{\mathrm{ST}}$ of all

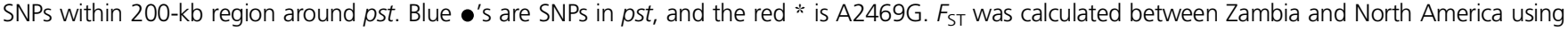
published genome sequences (see text). (B and C) The breakdown of EHH over distance between the derived (resistant) allele of the core SNP A2469G and SNPs within the distance of 1000 bases from the mutation are shown. Red line and blue line are EHH breakdown upstream and downstream of SNP A2469G, respectively. The gray points are a null distribution generated by calculating the EHH using other SNPs that are a similar frequency in the region as the core. The orange dash line indicates top $5 \% \mathrm{EHH}$ value of this null distribution while green dash line indicates median $\mathrm{EHH}$.

(Kraaijeveld and Godfray 1997; McKean et al. 2008). It is possible that costs may not be detected if they are measured in the "wrong" environment or the trait affected is not measured-for example, it may increase susceptibility to other pathogen genotypes.

The function and identity of viral restriction factors in invertebrates remains poorly understood, and the mechanism by which Pastrel protects flies against DCV is unknown. This contrasts with vertebrates where a diverse range of restriction factors have been characterized that inhibit all steps of viral infection (see Yan and Chen 2012 for review). Studying natural variation in susceptibility to viral infection is proving a powerful way to identify novel restriction factors in Drosophila (Bangham et al. 2008; Magwire et al. 2011; Cao et al. 2016), and future work on these genes is likely to provide new insights into how invertebrates defend themselves against infection. One clue as to the function of
Pastrel comes from its localization to lipid droplets in the larval fat body (Beller et al. 2006), as lipid droplets and lipid metabolism frequently play key roles in the viral replication cycle (Stapleford and Miller 2010). An alternative explanation is the reported involvement of Pastrel in the secretory pathway and Golgi organization (Bard et al. 2006).

We conclude that a single gene, pastrel, is the dominant factor that determines the susceptibility of $D$. melanogaster to DCV. This is a complex locus, with multiple alleles conferring different levels of resistance, with polymorphisms affecting both the expression and protein sequence of pastrel altering susceptibility to DCV. This gene has not been the target of strong directional selection, and the variation may be maintained by balancing selection. Overall, despite a single gene explaining most of the genetic variance in DCV susceptibility, this locus is remarkably complex. 


\section{Acknowledgments}

Thanks to Professor Jean-Luc Imler for helpful discussions, and Yuk-Sang Chan for embryo micro-injection. This work was supported by the European Research Council (281668 to F.M.J.) and Natural Environment Research Council (NE/L004232/1 to F.M.J.). C.C.'s PhD was funded by the Cambridge Commonwealth, European and International Trust and China Scholarship Council. R.C. is founded by the São Paulo Research Foundation (Fundação de Amparo à Pesquisa do Estado de São Paulo), grant no. 2015/08307-3 and 2013/25991-0.

\section{Literature Cited}

Alonso-Blanco, C., and B. Mendez-Vigo, 2014 Genetic architecture of naturally occurring quantitative traits in plants: an updated synthesis. Curr. Opin. Plant Biol. 18: 37-43.

Ashe, A., T. Belicard, J. Le Pen, P. Sarkies, L. Frezal et al., 2013 A deletion polymorphism in the Caenorhabditis elegans RIG-I homolog disables viral RNA dicing and antiviral immunity. Elife 2: e00994.

Bangham, J., S. A. Knott, K. W. Kim, R. S. Young, and F. M. Jiggins, 2008 Genetic variation affecting host-parasite interactions: major-effect quantitative trait loci affect the transmission of sigma virus in Drosophila melanogaster. Mol. Ecol. 17: 3800-3807.

Bard, F., L. Casano, A. Mallabiabarrena, E. Wallace, K. Saito et al., 2006 Functional genomics reveals genes involved in protein secretion and Golgi organization. Nature 439: 604-607.

Beller, M., D. Riedel, L. Jansch, G. Dieterich, J. Wehland et al., 2006 Characterization of the Drosophila lipid droplet subproteome. Mol. Cell. Proteomics 5: 1082-1094.

Bischof, J., R. K. Maeda, M. Hediger, F. Karch, and K. Basler, 2007 An optimized transgenesis system for Drosophila using germ-line-specific phiC31 integrases. Proc. Natl. Acad. Sci. USA 104: 3312-3317.

Burgner, D., S. E. Jamieson, and J. M. Blackwell, 2006 Genetic susceptibility to infectious diseases: big is beautiful, but will bigger be even better? Lancet Infect. Dis. 6: 653-663.

Cao, C., M. M. Magwire, F. Bayer, and F. M. Jiggins, 2016 A polymorphism in the processing body component Ge-1 controls resistance to a naturally occurring Rhabdovirus in Drosophila. PLoS Pathog. 12: e1005387.

Carpenter, J. A., J. D. Hadfield, J. Bangham, and F. M. Jiggins, 2012 Specific interactions between host and parasite genotypes do not act as a constraint on the evolution of antiviral resistance in Drosophila. Evolution 66: 1114-1125.

Charlesworth, D., 2006 Balancing selection and its effects on sequences in nearby genome regions. PLoS Genet. 2: e64.

Christian, P. D., 1987 Studies on Drosophila C and A viruses in Australian populations of Drosophila melanogaster. Ph.D. Thesis, Australian National University, Canberra, Australia.

Chtarbanova, S., O. Lamiable, K. Z. Lee, D. Galiana, L. Troxler et al., 2014 Drosophila C virus systemic infection leads to intestinal obstruction. J. Virol. 88: 14057-14069.

Cogni, R., C. Cao, J. P. Day, C. Bridson, and F. M. Jiggins, 2016 The genetic architecture of resistance to virus infection in Drosophila. Mol. Ecol. 25: 5228-5241.

Contamine, D., A. M. Petitjean, and M. Ashburner, 1989 Genetic resistance to viral infection: the molecular cloning of a Drosophila gene that restricts infection by the rhabdovirus sigma. Genetics 123: 525-533.

Cordes, E. J., K. D. Licking-Murray, and K. A. Carlson, 2013 Differential gene expression related to Nora virus infection of Drosophila melanogaster. Virus Res. 175: 95-100.
Danecek, P., A. Auton, G. Abecasis, C. A. Albers, E. Banks et al., 2011 The variant call format and VCFtools. Bioinformatics 27: 2156-2158.

Dubnau, J., A. S. Chiang, L. Grady, J. Barditch, S. Gossweiler et al., 2003 The staufen/pumilio pathway is involved in Drosophila long-term memory. Curr. Biol. 13: 286-296.

Egea, R., S. Casillas, and A. Barbadilla, 2008 Standard and generalized McDonald-Kreitman test: a website to detect selection by comparing different classes of DNA sites. Nucleic Acids Res. 36: W157-W162.

Faria, V. G., N. E. Martins, T. Paulo, L. Teixeira, E. Sucena et al., 2015 Evolution of Drosophila resistance against different pathogens and infection routes entails no detectable maintenance costs. Evolution 69: 2799-2809.

Ferreira, A. G., H. Naylor, S. S. Esteves, I. S. Pais, N. E. Martins et al., 2014 The Toll-dorsal pathway is required for resistance to viral oral infection in Drosophila. PLoS Pathog. 10: e1004507.

Gautier, M., and R. Vitalis, 2012 rehh: an R package to detect footprints of selection in genome-wide SNP data from haplotype structure. Bioinformatics 28: 1176-1177.

Grenier, J. K., J. R. Arguello, M. C. Moreira, S. Gottipati, J. Mohammed et al., 2015 Global diversity lines - a five-continent reference panel of sequenced Drosophila melanogaster strains. G3 (Bethesda) 5: 593-603.

Hadfield, J. D., 2010 MCMC methods for multi-response generalized linear mixed models: the MCMCglmm R package. J. Stat. Softw. 33: 1-22.

Hedges, L. M., and K. N. Johnson, 2008 Induction of host defence responses by Drosophila C virus. J. Gen. Virol. 89: 1497-1501.

Hill, A. V. S., 2012 Evolution, revolution and heresy in the genetics of infectious disease susceptibility. Philos. Trans. R. Soc. Lond. B Biol. Sci. 367: 840-849.

Jiggins, F. M., and M. C. Tinsley, 2005 An ancient mitochondrial polymorphism in Adalis bipunctata linked to a sex-ratio-distorting bacterium. Genetics 171: 1115-1124.

Johnson, K. N., and P. D. Christian, 1998 The novel genome organization of the insect picorna-like virus Drosophila C virus suggests this virus belongs to a previously undescribed virus family. J. Gen. Virol. 79: 191-203.

Jousset, F. X., N. Plus, G. Croizier, and M. Thomas, 1972 Existence in Drosophila of 2 groups of picornavirus with different biological and serological properties. C. R. Acad. Sci. Hebd. Seances Acad. Sci. D 275: 3043-3046.

Kemp, C., S. Mueller, A. Goto, V. Barbier, S. Paro et al., 2013 Broad RNA interference-mediated antiviral immunity and virus-specific inducible responses in Drosophila. J. Immunol. 190: 650-658.

King, E. G., S. J. Macdonald, and A. D. Long, 2012a Properties and power of the Drosophila synthetic population resource for the routine dissection of complex traits. Genetics 191: 935-949.

King, E. G., C. M. Merkes, C. L. McNeil, S. R. Hoofer, S. Sen et al., 2012b Genetic dissection of a model complex trait using the Drosophila synthetic population resource. Genome Res. 22: 1558-1566.

King, E. G., B. J. Sanderson, C. L. McNeil, A. D. Long, and S. J. Macdonald, 2014 Genetic dissection of the Drosophila melanogaster female head transcriptome reveals widespread allelic heterogeneity. PLoS Genet. 10: e1004322.

Kraaijeveld, A. R., and H. C. Godfray, 1997 Trade-off between parasitoid resistance and larval competitive ability in Drosophila melanogaster. Nature 389: 278-280.

Laurie, C. C., and L. F. Stam, 1994 The effect of an intronic polymorphism on alcohol dehydrogenase expression in Drosophila melanogaster. Genetics 138: 379-385.

Librado, P., and J. Rozas, 2009 DnaSP v5: a software for comprehensive analysis of DNA polymorphism data. Bioinformatics 25: 1451-1452. 
Lischer, H. E., and L. Excoffier, 2012 PGDSpider: an automated data conversion tool for connecting population genetics and genomics programs. Bioinformatics 28: 298-299.

Longdon, B., L. Wilfert, and F. M. Jiggins, 2012 The Sigma viruses of Drosophila. Caister Academic Press, Poole, United Kingdom.

Longdon, B., C. Cao, J. Martinez, and F. M. Jiggins, 2013 Previous exposure to an RNA virus does not protect against subsequent infection in Drosophila melanogaster. PLoS One 8: e73833.

Longdon, B., M. A. Brockhurst, C. A. Russell, J. J. Welch, and F. M. Jiggins, 2014 The evolution and genetics of virus host shifts. PLoS Pathog. 10: e1004395.

Mackay, T. F., S. Richards, E. A. Stone, A. Barbadilla, J. F. Ayroles et al., 2012 The Drosophila melanogaster genetic reference panel. Nature 482: 173-178.

Magwire, M. M., F. Bayer, C. L. Webster, C. Cao, and F. M. Jiggins, 2011 Successive increases in the resistance of Drosophila to viral infection through a transposon insertion followed by a duplication. PLoS Genet. 7: e1002337.

Magwire, M. M., D. K. Fabian, H. Schweyen, C. Cao, B. Longdon et al., 2012 Genome-wide association studies reveal a simple genetic basis of resistance to naturally coevolving viruses in Drosophila melanogaster. PLoS Genet. 8: e1003057.

Martins, N. E., V. G. Faria, V. Nolte, C. Schlotterer, L. Teixeira et al., 2014 Host adaptation to viruses relies on few genes with different cross-resistance properties. Proc. Natl. Acad. Sci. USA 111: 5938-5943.

McDonald, J. H., and M. Kreitman, 1991 Adaptive protein evolution at the Adh locus in Drosophila. Nature 351: 652-654.

McKean, K. A., C. P. Yourth, B. P. Lazzaro, and A. G. Clark, 2008 The evolutionary costs of immunological maintenance and deployment. BMC Evol. Biol. 8: 76.

Obbard, D. J., and G. Dudas, 2014 The genetics of host-virus coevolution in invertebrates. Curr. Opin. Virol. 8: 73-78.

Obbard, D. J., J. J. Welch, K. W. Kim, and F. M. Jiggins, 2009 Quantifying adaptive evolution in the Drosophila immune system. PLoS Genet. 5: e1000698.

Pool, J. E., R. B. Corbett-Detig, R. P. Sugino, K. A. Stevens, C. M. Cardeno et al., 2012 Population genomics of sub-saharan Drosophila melanogaster: African diversity and non-African admixture. PLoS Genet. 8: e1003080.

R Development Core Team, 2011 R: A Language and Environment for Statistical Computing. R Foundation for Statistical Computing, Vienna, Austria.

Reed, L. J. M. H., 1938 A simple method of estimating fifty per cent endpoints. Am. J. Hyg. 27: 493-497.

Routtu, J., and D. Ebert, 2015 Genetic architecture of resistance in Daphnia hosts against two species of host-specific parasites. Heredity (Edinb) 114: 241-248.

Sabeti, P. C., E. Walsh, S. F. Schaffner, P. Varilly, B. Fry et al., 2005 The case for selection at CCR5-Delta32. PLoS Biol. 3: e378.

Shin, J-H., S. Blay, J. Graham, and B. McNeney, 2006 LDheatmap: an R function for graphical display of pairwise linkage disequilibria between single nucleotide polymorphisms. J Stat Soft 16: Code Snippet 3.

Schmidt, J. M., R. T. Good, B. Appleton, J. Sherrard, G. C. Raymant, M. R. Bogwitz, J. Martin, P. J. Daborn, M. E. Goddard, P. Batterham, and C. Robin, 2010 Copy number variation and transposable elements feature in recent, ongoing adaptation at the Cyp6g1 locus. PLoS Genet 6: e1000998. doi: 10.1371/journal.pgen.1000998

Smith, N. G., and A. Eyre-Walker, 2002 Adaptive protein evolution in Drosophila. Nature 415: 1022-1024.

Stapleford, K. A., and D. J. Miller, 2010 Role of cellular lipids in positive-sense RNA virus replication complex assembly and function. Viruses 2: 1055-1068.

Teixeira, L., A. Ferreira, and M. Ashburner, 2008 The bacterial symbiont Wolbachia induces resistance to RNA viral infections in Drosophila melanogaster. PLoS Biol. 6: e2.

Unckless, R. L., V. M. Howick, and B. P. Lazzaro, 2016 Convergent balancing selection on an antimicrobial peptide in Drosophila. Curr. Biol. 26: 257-262.

Venken, K. J., Y. He, R. A. Hoskins, and H. J. Bellen, 2006 P[acman]: a BAC transgenic platform for targeted insertion of large DNA fragments in D. melanogaster. Science 314: 1747-1751.

Venken, K. J., J. W. Carlson, K. L. Schulze, H. Pan, Y. He et al., 2009 Versatile P[acman] BAC libraries for transgenesis studies in Drosophila melanogaster. Nat. Methods 6: 431-434.

Warming, S., N. Costantino, D. L. Court, N. A. Jenkins, and N. G. Copeland, 2005 Simple and highly efficient BAC recombineering using galK selection. Nucleic Acids Res. 33: e36.

Webster, C. L., F. M. Waldron, S. Robertson, D. Crowson, G. Ferrari et al., 2015 The discovery, distribution, and evolution of viruses associated with Drosophila melanogaster. PLoS Biol. 13: e1002210.

Wilfert, L., and F. M. Jiggins, 2013 The dynamics of reciprocal selective sweeps of host resistance and a parasite counteradaptation in Drosophila. Evolution 67: 761-773.

Yampolsky, L. Y., C. T. Webb, S. A. Shabalina, and A. S. Kondrashov, 1999 Rapid accumulation of a vertically transmitted parasite triggered by relaxation of natural selection among hosts. Evol. Ecol. Res. 1: 581-589.

Yan, N., and Z. J. Chen, 2012 Intrinsic antiviral immunity. Nat. Immunol. 13: 214-222.

Ye, K., M. H. Schulz, Q. Long, R. Apweiler, and Z. Ning, 2009 Pindel: a pattern growth approach to detect break points of large deletions and medium sized insertions from paired-end short reads. Bioinformatics 25: 2865-2871.

Zeng, K., S. Mano, S. Shi, and C. I. Wu, 2007 Comparisons of siteand haplotype-frequency methods for detecting positive selection. Mol. Biol. Evol. 24: 1562-1574.

Zhu, F., H. Ding, and B. N. Zhu, 2013 Transcriptional profiling of Drosophila S2 cells in early response to Drosophila C virus. Virol. J. 10: 210 